\title{
Incidences, Trends and Long Term Outcomes of Retinoblastoma in Three Cancer Registries, Thailand
}

\author{
Piyathida Wongmas ${ }^{1}$, Arunee Jetsrisuparb ${ }^{1}$, Patcharee Komvilaisak ${ }^{1}$, Krittika \\ Suwanrungruang ${ }^{2}$, Worawut Choeyprasert ${ }^{3}$, Hutcha Sriplung ${ }^{4}$, Surapon \\ Wiangnon ${ }^{1 *}$
}

\begin{abstract}
Background: Retinoblastoma (RB) is rare, albeit the most common primary intraocular malignancy among children. To elucidate the true incidence, trend and survival, we studied incidences and trends of retinoblastoma in a large population with long-term follow-up using data from 3 population-based cancer registries. Objective: To describe the incidence, trends and survival of RB between 1990 and 2009 in Khon Kaen, Songkhla and Chiang Mai, Thailand. Materials and Methods: We sourced the data from the cancer registries in Khon Kaen, Songkhla and Chiang Mai on children with retinoblastoma, diagnosed between 1990 and 2009. Retinoblastoma was defined as per the International Classification of Disease for Oncology version 3 using the code 9510/3. Incidence was analyzed using the standard method with the criteria of the International Association of Cancer Registries. The Kaplan-Meier method was applied to calculate cumulative survival. Trends were calculated using the $\log$ rank test. Results: We identified 75 cases of children between 0 and 15 years of age diagnosed with RB (Khon Kaen 31, Chiang Mai 20, Songkhla 24). Males and females were equally affected. The most common age group was 0-4 years. The morphological verification of the disease was $90.7 \%$. The respective ASR in Khon Kaen, Chiang Mai and Songkhla was 4.4, 4.0 and 4.6 per million; for which the overall ASR for all 3 areas was 4.3 per million. The respective trend in incidence was 4, 2.8, 5.8 and 5.4 during 1990-4, 1995-9, 2000-4 and 2005-9. Overall, incidence trended gradually upward by $2 \%$ annually. The respective survival rate in Khon Kaen, Chiang Mai and Songkhla was 50,40 and $75 \%$ (differences not significantly different at $\mathrm{p}=\mathbf{0 . 1 4}$ ) and the overall survival for all centers was $60 \%$. Conclusions: Over the last two decades, the incidence and overall survival of retinoblastoma has increased. The ASRs and survival in Thailand were less than those in resource-rich countries.
\end{abstract}

Keywords: Retinoblastoma - incidence - trend - survival - Thailand

Asian Pac J Cancer Prev, 16 (16), 6899-6902

\section{Introduction}

Retinoblastoma $(\mathrm{RB})$ is the most common primary intraocular malignancy of childhood. According to a report by the Thai Pediatric Oncology Group, it is the 7th most common cancer in childhood with an incidence of 3.1 per million; with 35-40 new cases each year (Wiangnon et al., 2011). More than $90 \%$ of all cancers are diagnosed in younger children under 5 . The average age at diagnosis is 18 months (Broaddus et al., 2009). During the past two decades, the incidence among children with retinoblastoma increased; however, advances in therapeutic strategies resulted in improved survival (Chen, 2010).

Thai Pediatric Oncology Group (ThaiPOG) reported on the incidence and survival of children with retinoblastoma between 2003 and 2005. It was a relatively short study so long term trends could not be evaluated. We, therefore, set out to analyze incidence, trends and long-term survival characteristics of children with retinoblastoma between 1990 and 2009, using the population-based data from cancer registries in Khon Kaen, Chiang Mai, and Songkhla.

\section{Materials and Methods}

Retinoblastoma cases, between 0-15 years of age, diagnosed between January 1990 and December 2009 were retrieved from the population-based data in the cancer registries of Khon Kaen, Songkhla and Chiang Mai provinces, which are located in the northeast, south and north of Thailand, respectively (Figure 1). The medical records of retinoblastoma cases at Srinagarind Hospital (Khon Kaen Cancer Registry, KKCR), Songklanagarind Hospital (Songkhla Cancer Registry, SKCR) and Maharaj

${ }^{1}$ Department of Pediatrics, ${ }^{2}$ Cancer Unit, Faculty of Medicine, Khon Kaen University, Khon Kaen, ${ }^{3}$ Department of Pediatrics, Faculty of Medicine, Chiang Mai University, Chiang Mai, ${ }^{4}$ Epidemiology Unit, Faculty of Medicine, Prince of Songkhla University, Songkhla, Thailand*For correspondence: suraponwiangnon@gmail.com 


\section{Piyathida Wongmas et al}

Hospital (Chiang Mai Cancer Registry, CMCR) were reviewed for the information required to do our analyses.

All of the registries employ international data standards. Retinoblastoma was recorded under the code 9510/3, according to International Classification of Disease for Oncology, version 3 (Fritz et al., 2000). Metastatic cancer to the eye was excluded. Patient status was confirmed using the individual's identification number in the Statistical Data Bank and Information Dissemination Division of National Statistic Office, (National Statistic Office, 2003). The date censored was December 31, 2009.

All demographic data including sex, age at diagnosis, basis of diagnosis, morphology, laterality, treatment strategy and status of patient were collected. The patients

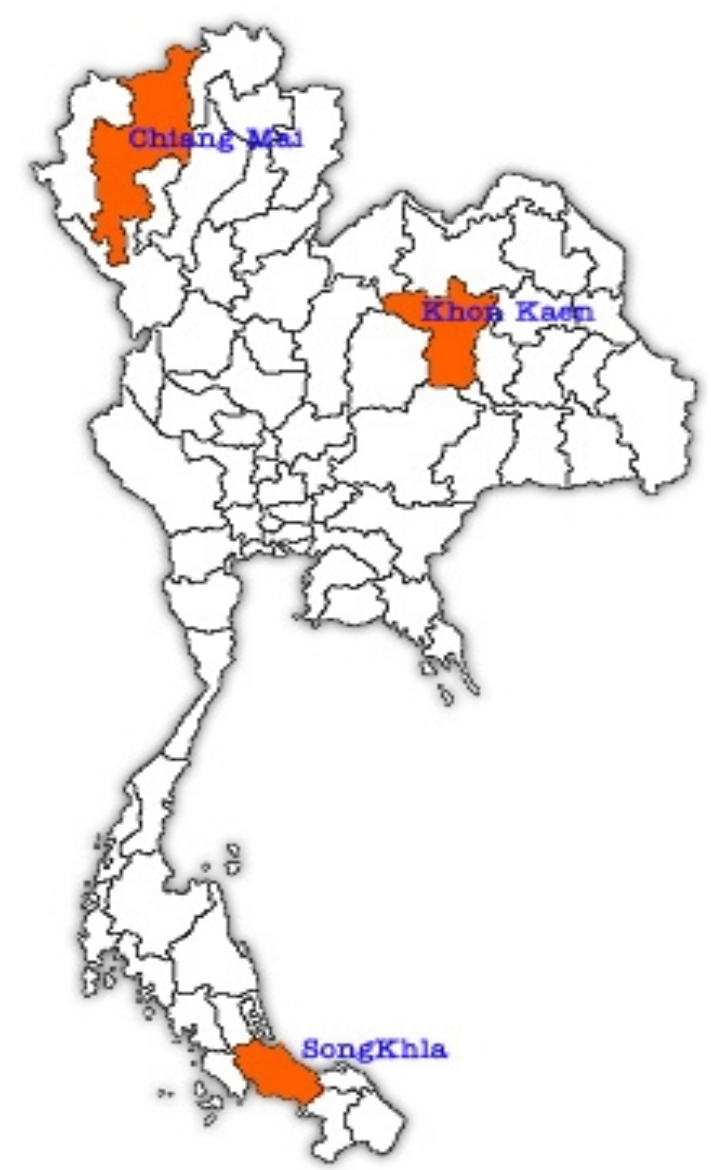

Figure 1. Map of Thailand, Highlighting Chiang Mai, Khon Kaen and Songkhla Province

Table 1. Characteristics of Retinoblastoma Patients

\begin{tabular}{lccc}
\hline Characteristic & \multicolumn{3}{c}{ Number of cases } \\
& Male (\%)] & Female $(\%)$ & Total (\%) \\
\hline Sex & $37(49.3)$ & $38(50.7)$ & $75(100)$ \\
Age & & & \\
0-4 years & $36(46.2)$ & $37(46.4)$ & $70(93.3)$ \\
5-9 years & $3(3.8)$ & $1(1.8)$ & $4(5.3)$ \\
10-15 years & - & $1(1.8)$ & $1(1.4)$ \\
Laterality & & & \\
$\quad$ Right eye & $9(11.5)$ & $8(10.3)$ & $16(21.3)$ \\
$\quad$ Left eye & $13(16.7)$ & $12(15.4)$ & $24(32.0)$ \\
$\quad$ Bilateral & $9(11.5)$ & $9(11.5)$ & $17(22.7)$ \\
$\quad$ Unknown & $8(10.3)$ & $10(12.8)$ & $18(24.0)$ \\
\hline
\end{tabular}

not residing in Khon Kaen, Chiang Mai and Songkhla were excluded.

The age-standardized population incidence rate was calculated as per the standard method of the International Association of Cancer Registry (Parkin, 1998). The children population denominator was estimated using the 2000 Population and Housing Census (National Statistic Office, 2003). The survival rate was analyzed using the Kaplan-Meier method. Incidence trends were calculated as per the annual percent change while trends of survival were calculated using the log rank test.

\section{Results}

Between 1990 and 2009, there were 75 cases diagnosed as having retinoblastoma in the three registries (24 cases from KKCR, 31 cases from CMCR and 20 cases from CMCR). Males and females were affected equally ( $\mathrm{N}=37$ and 38). The most common age groups were infants and toddlers. Most of the cases were diagnosed before 2 years of age. The respective mean and median age at diagnosis was 1.96 years (SD 1.87) and 2 years (range, 1 month to 12 years). The diseases were unilateral more than bilateral $(\mathrm{N}=40$ and 17$)$ while 18 patients had unknown laterality. (Table1)

Table 2. Basis of Diagnosis and Treatment Modality

\begin{tabular}{lr}
\hline & Number of cases (\%) \\
\hline Basis of diagnosis & $1(1.3)$ \\
History and physical exam & $5(6.7)$ \\
Radiology & $1(1.3)$ \\
Histology of metastasis & $67(89.4)$ \\
Histology of primary tumor & $1(1.3)$ \\
Unknown & 90.7 \\
\% Morphological verification (MV)* & \\
Treatment modality & $7(9.0)$ \\
Surgery & $4(5.1)$ \\
Chemotherapy & $1(1.3)$ \\
Radiation & $26(33.3)$ \\
Surgery and chemotherapy & $10(12.8)$ \\
Surgery and radiation & $1(1.3)$ \\
Radiation and chemotherapy & $21(27.0)$ \\
Surgery and radiation and chemotherapy & $3(3.8)$ \\
Refuse treatment & $5(6.4)$ \\
Unknown &
\end{tabular}

*MV = histology of primary and metastasis

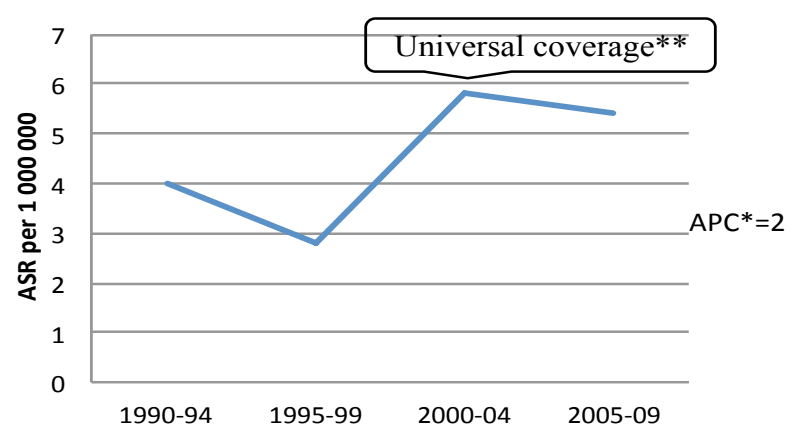

Figure 2. Overall ASR; *APC,Annual Percent Change; ** Universal coverage, Universal Coverage Health Scheme in Thailand, Started in 2002 


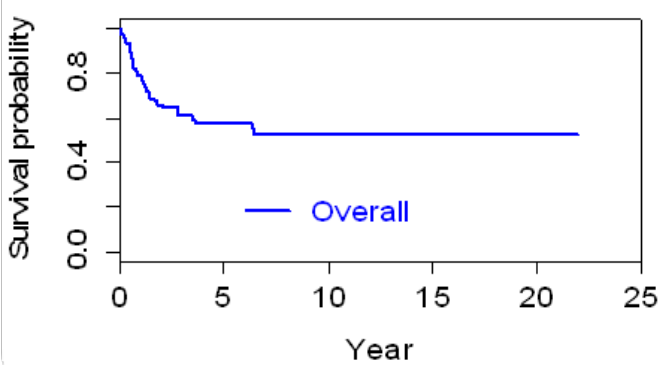

Figure 3. Survival Curve for Retinoblastoma Patients at the 3 Registries (Khon Kaen, Songkhla, Chiang Mai), 1990-2009

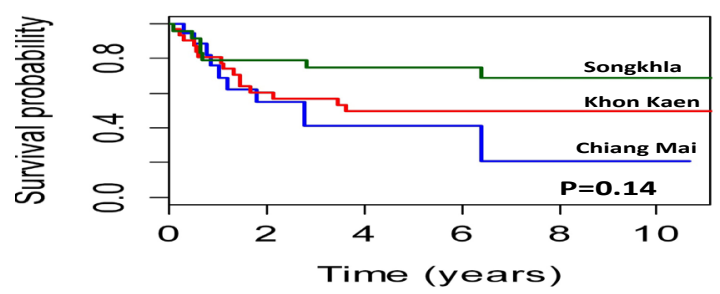

Figure 4. Survival Curve for Retinoblastoma Patients at the 3 Registries (Khon Kaen, Songkhla, Chiang Mai), 1990-2009

Sixty-seven patients were diagnosed based on histology of the primary tumor, while 1 was diagnosed based on histology of the metastasis. The morphological verification was $90.7 \%$. (Table 2)

Most of the patients were treated with multiple modalities, namely: surgery, chemotherapy and radiation. Three patients refused treatment while the treatment data for 5 patients were not defined. (Table 2)

\section{Incidence and trend}

The respective ASRs at 5-year intervals between 19902009 in Khon Kaen, Chiang Mai and Songkhla ranged from 1.1 to 6.6 per million, 2.4 to 6 per million and 2.4 to 6 per million (differences not statistically significant: $\mathrm{p}=0.17$ ). The overall ASRs for all three centers at 5-year intervals ranged from 2.8 to 5.8 per million. (Table 3 ) The incidence of bilateral retinoblastoma was 1.0 per million.

Incidence generally trended upward: the calculated annual percent change was $2 \%$ per year. (Figure 2 )

\section{Survival}

The survival rates for each cancer registry varied but the differences are not statistically significant (Khon Kaen, Chiang Mai, Songkhla: 50\%, 75\%, 40\%, p=0.14). The overall survival rate for all cases at the 3 registries was $60 \%$. (Figure 3,4 ) Survival trended to increase by $1 \%(\mathrm{p}=0.41)$.

\section{Discussion}

Even though retinoblastoma (RB) is the most common primary intraocular tumor in childhood, it is a rare disease. The prognosis is good, especially for those patients at an early stage of the disease (Augsburger, 2009; Kaufman, 2014; Friedman, 2011). A large population study to determine the true incidence in Thailand was needed.
We, therefore, investigated the incidence and long-term outcomes, using data from three long-standing cancer registries in Thailand.

According to the ThaiPOG study, $3.4 \%$ of childhood cancers are RB, with an incidence of 3.1 per million in Thailand (Wiangnon et al., 2011). The peak incidence was in the infant and toddler groups (MacCarthy et al., 2006). Interestingly, children over 4 years of age comprised $6.7 \%$ of cases.

Retinoblastoma occurs in two forms, heritable and non-heritable. The heritable form includes bilateral RB and a small number of children with unilateral tumors (Friedman, 2011): in general, the incidence is similar internationally. The proportion of bilateral RB in the current study comprises $22.7 \%$, which was lower than the incidence reported in the USA (26.7\%) (Broaddus, 2009) and Great Britain (36.3\%) (MacCarthy, 2009). Total incidence is generally higher in developing countries, especially in sub-Sahan Africa (Stiller, 2004). As in our study, the ASRs in other Asian countries range from 1 to 5 per million except in Singapore (i.e., 1 to 5 in Taiwan (Chen et al., 2010); 3.1 in Thailand (Wiangnon et al, 2011); 4.4 in Japan (Stiller, 2004); and 11.1 in Singapore (Saw, 2000). The incidences were, however, less than those in Europe and USA (Ries et al., 1999; MacCarthy et al., 2006).

The incidence of RB in Thailand may have been under-registered. After implementing universal health coverage in Thailand in 2002 (Tangcharoensathien, 2010), the incidence rate significantly increased; perhaps because more poor people are getting access to health services and more people are being referred to centers which can verify the diagnosis. With better health coverage and associated reporting, incidence has been trending upward by 2 percent annually.

The incidence at each center in this study was not significantly different but it did vary. The lowest survival was observed in Chiang Mai province where people living in remote mountainous areas might have difficulty accessing treatment. There are, moreover, some hill-tribes who might have beliefs opposing modern treatment. The distribution of disease staging and details of treatment modalities could not be defined using this type of population-based data from the cancer registries center.

The survival rates of RB in the current study were generally less than of those in developed and resourcerich countries (Young et al., 1999, Seregard et al., 2004; Broaddus et al., 2009). In general, advances in diagnostic methods and treatment modalities have improved incidence rates and treatment outcomes (Chen, 2010). Survival trends in the current study increased $1 \%$ overall $(\mathrm{p}=0.41)$.

Due to outdated data recording protocols at the 3 registries, some older registrations had no individual number and it was not, therefore, possible to thoroughly evaluate the patient's status. In addition, specific or detailed information are not recorded in a populationbased registry; consequently, survival could not be assessed by important risk factors of disease; such as, staging and genetic determinants.

In conclusion, during the past two decades, the 
Piyathida Wongmas et al

incidence and overall survival of retinoblastoma were increased. The ASRs and survival in Thailand were less than those in resource-rich countries; however, outcomes are improving year by year.

\section{Acknowledgements}

The authors thank (a) the staff at the Cancer Registries at Khon Kaen, Chiang Mai and Songkhla for providing the data and (b) Mr. Bryan Roderick Hamman for assistance with the English-language presentation.

\section{References}

Augsburger JJ (2009). Retinoblastoma. In: Yanoff and Duker. Ophthalmology. 3rd ed. Missouri: Mosby, 887-94.

Broaddus E, Topham A, Singh DA (2009). Incidence of retinoblastoma in the USA: 1975-2004. Br J Ophthalmol, 93, 21-23.

Broaddus E, Topham A, Singh DA (2009). Survival with retinoblastoma in the USA: 1975-2004. Br J Ophthalmol, 93, 24-27.

Chen YH, Lin YH, Hsu WM, Lee SM, Cheng CY (2010). Retinoblastoma in Taiwan: incidence and survival characteristics from 1979 to 2003 . Eye (Lond), 24, 318-22.

Friedman DL (2011). Retinoblastoma. In: Lanzkowsky P. Manual of pediatric hematology and oncology. 5th ed. Amsterdam: Academic Pr, 759-75.

Fritz A, Percy C, Jack A, Shanmugaratnam K, Sobin L, Parkin D, Whelan S (2000). International Classification of Disease for Oncology, 3rd edition. World Health Organization, Geneva.

Kaufman PL, Paysse EA (2014). Overview of retinoblastoma [Internet]. [cited 2014 Jul 18]; Available from: http://goo. $\mathrm{gl} / \mathrm{kt} 2 \mathrm{~B} 9 \mathrm{X}$

MacCarthy A, Birch JM, Draper GJ, et al (2009). Retinoblastoma in Great Britain 1963-2002. Br J Ophthalmol, 93, 33-37.

MacCarthy A, Drapera GJ, Steliarova-Foucher E, Kingstone JE (2006). Retinoblastoma incidence and survival in European children (1978-1997). Report from the automated childhood cancer information system project. Eur J Cancer, 42, 2092102.

Naseripour M, Falavarjani GK, Bakhtiari P, Vosough P, Aryan F (2009). Retinoblastoma survival in Iran: 10-year experiences of a referral center. Iranian J Ophthalmol, 21, 17-24.

Naseripour M, Nazari H, Bakhtiari P, Modarres-zadeh M, Vosough P, Ausari M (2009). Retinoblastoma in Iran: outcomes in terms of patients' survival and globe survival. Br J Ophthalmol, 93, 28-32.

National Statistic Office (2003). Statistical data bank and information dissemination division. The 2000 population and housing census. Office of prime minister.

Parkin DM, Kramarova E, Draper GL, et al (1998). International incidence of childhood cancer, Vol II. IARC scientific publication, 144.

Ries LG, Smith MA, Gurney, et al (1999). Cancer incidence and survival among children and adolescent: United States SEER Program 1975-1995. NIH. Pub. No. 99-4649: Bethesda.

Seregard S, Lundell G, Svedberg H, Kivela T (2004). Incidence of retinoblastoma from 1958 to 1998 in Northern Europe: advantages of birth cohort analysis. Am Acad Ophthalmol, 111, 1228-32.

Tamboli A, Podgor MJ, Horm JW (1990). The incidence of retinoblastoma in the United States: 1974 through 1985. Arch Ophthalmol, 108, 128-32.

Tangcharoensathien V, Swasdiworn W, Jongudomsuk P, et al (2010). Universal coverage scheme in Thailand: equity outcomes and future agendas to meet challenges. Background paper, 43; World health report, World Health Organization.

Saw SM, Tan N, Lee SB, Au Eong KG, Chia KS (2000). Incidence and survival characteristics of retinoblastoma in Singapore from 1968-1995. J Pediatr Ophthalmol Strabismus, 37, 87-93.

Stiller CA (2004). Epidemiology and genetics of childhood cancer. Oncogene, 23, 6429-44.

Wiangnon S, Veerakul G, Nuchprayoon I, et al. (2011). Childhood cancer incidence and survival 2003-2005, Thailand: study from the Thai pediatric oncology group. Asian Pac J Cancer Prev, 12, 2215-20.

Young JL, Smith MA, Rofferes SD, et al (1999). Retinoblastoma. In: Ries LAG, Smith MA, Gurney JG, Tamra T. Young JL, Bunin GR, editors. Cancer incidence and survival among children and adolescents: Unites States SEER Program 1975-1995. NIH Pub. No. 99-4649; 73-8. 